\title{
Design an Information Management System for Hospitals of Duhok City Using Android Application System
}

\author{
Hewa Majeed Zangana
}

Department of Computer Science / College of Computer Science and IT / Nawroz University / Kurdistan Region of Iraq

\begin{abstract}
The proposed system consists of two applications, we made both of them on android system by online, and we made this system especially for hospitals in Duhok governorate. The name of the application is (management hospital) that used for hospital management system, in order to input all information about hospitals, and changing the information too and we made another application which called (Hospital user), this application will be the one which people can use. They can see easily all information by regulation, and that information consist of (name of doctors, specialty of doctors, phone number, holiday days) and we used (rating) in order to evaluate the doctors and used (Search) for searching the name of doctors and epode is made by easier shape and regulation.
\end{abstract}

Keywords: Hospital, Management, Information, Regulation.

\section{INTRODUCTION}

A hospital offers considerable advantages for both patient and society. A number of health problems require intensive medical treatment and personal care, which normally cannot be available in a patient's home or in the clinic of the doctor. This is possible only in a hospital where a large number of professionally and technically skilled people apply their knowledge and skill with the help of world-class expertise, advanced sophisticated equipment and appliances. A hospital is a health care institution providing patient treatment with specialized medical and nursing staff and medical equipment. A district hospital typically is the major health care facility in its region, We this system making for management hospital and user this system consist of two applications. We made both of them on android system by on line, and we can this system for special hospital in Duhok governorate. The name of the application (management hospital) and that use for hospital management, to input all information about hospital, and changing the information too $\mathrm{m}$ and we made another application is called (Hospital user) this application which people can use. They can see easily all information by regulation, and that information consist of (name, doctor, specialty of doctor, phone number, holiday days) And we use (rating) how to evaluate the doctor and use (Search) for searching the name of doctor and epode is made by easier shape and regulation.

\section{PROPOSED SYSTEM}

In this section we will explain the design of the search, the server, how to link it with the application, the database, structure of the system is implemented step by step, explain each step, and how it works.

\subsection{Design}

The design section consists of two parts, including:

\subsubsection{Client Side}

The client-side part explains how to choose the programming language and mobile application, including:

\subsubsection{Choice of Programming Language}

Choosing a programming language depends on your language experience and the scope of the application you are building. While small applications are often created using only one language, it is not uncommon to develop large applications using multiple languages. The propose application to be built is not a web-based application that needs internet facilities to function but a standalone application. The choice of programming language to use for this program is Android Application. The structure of the Basic programming language is very simple, particularly as to the executable code. Android Application has many new and improved features such as inheritance, interfaces, and overloading that make it a powerful object-oriented programming language. It is particularly easy to develop graphical user interfaces and to connect them to handler functions provided by the application. 


\subsubsection{Server Side}

A single overall computation is distributed across multiple processes or devices. Servers can provide various functionalities, often called "services", such as sharing data or resources among multiple clients, or performing computation for a client. A single server can serve multiple clients, and a single client can use multiple servers. A client process may run on the same device or may connect over a network to a server on a different device. Typical servers are database servers, file servers, mail servers, print servers, web servers, game servers, and application servers. (en.wikipedia.org)

A customer sends a request to the server, which performs some action and sends a response back to the customer by php as shown in figure 3 .

\subsubsection{Web server}

Host web pages. a web server is what makes the World Wide Web possible. each website has one or more web servers as show in figure 1.

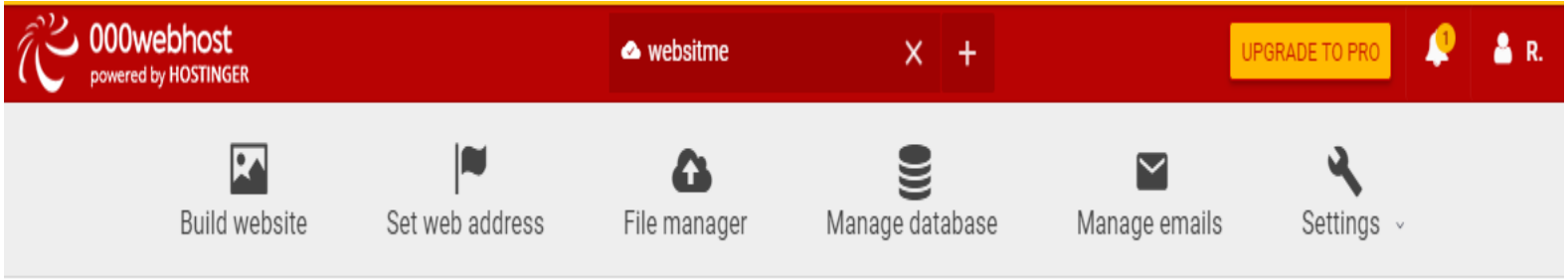

Figure 1: Web Server

\subsubsection{File Manager}

Share files and folders, storage space to hold files and folders, or both, over a network as show in figure 2.

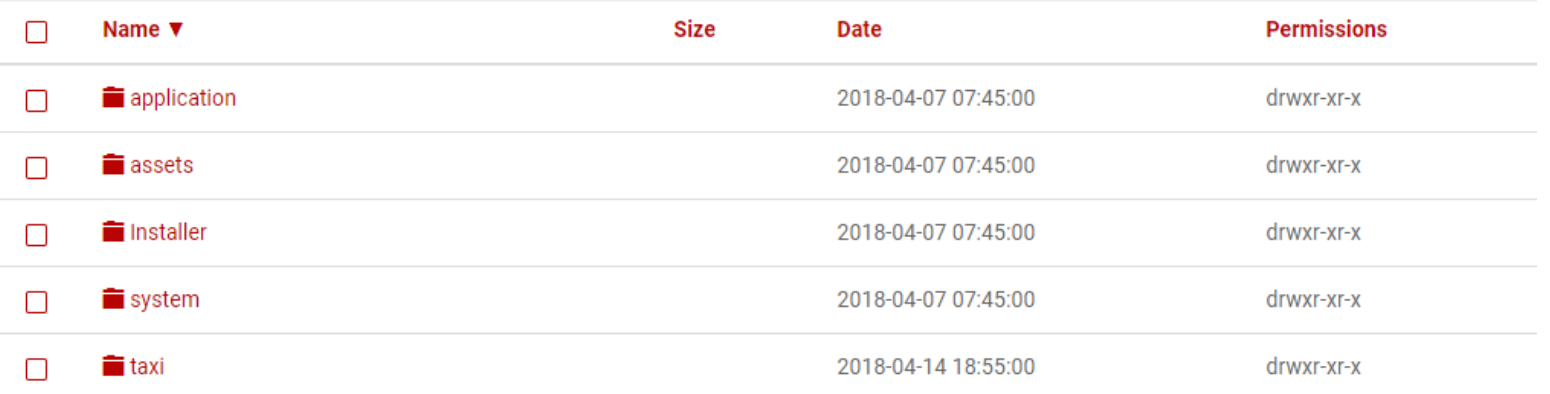

Figure 2: File Manager

\subsubsection{Database server}

The server Maintains and shares any form of database (organized collections of data with predefined properties that may be displayed in a table) over a network as show in figure 3.

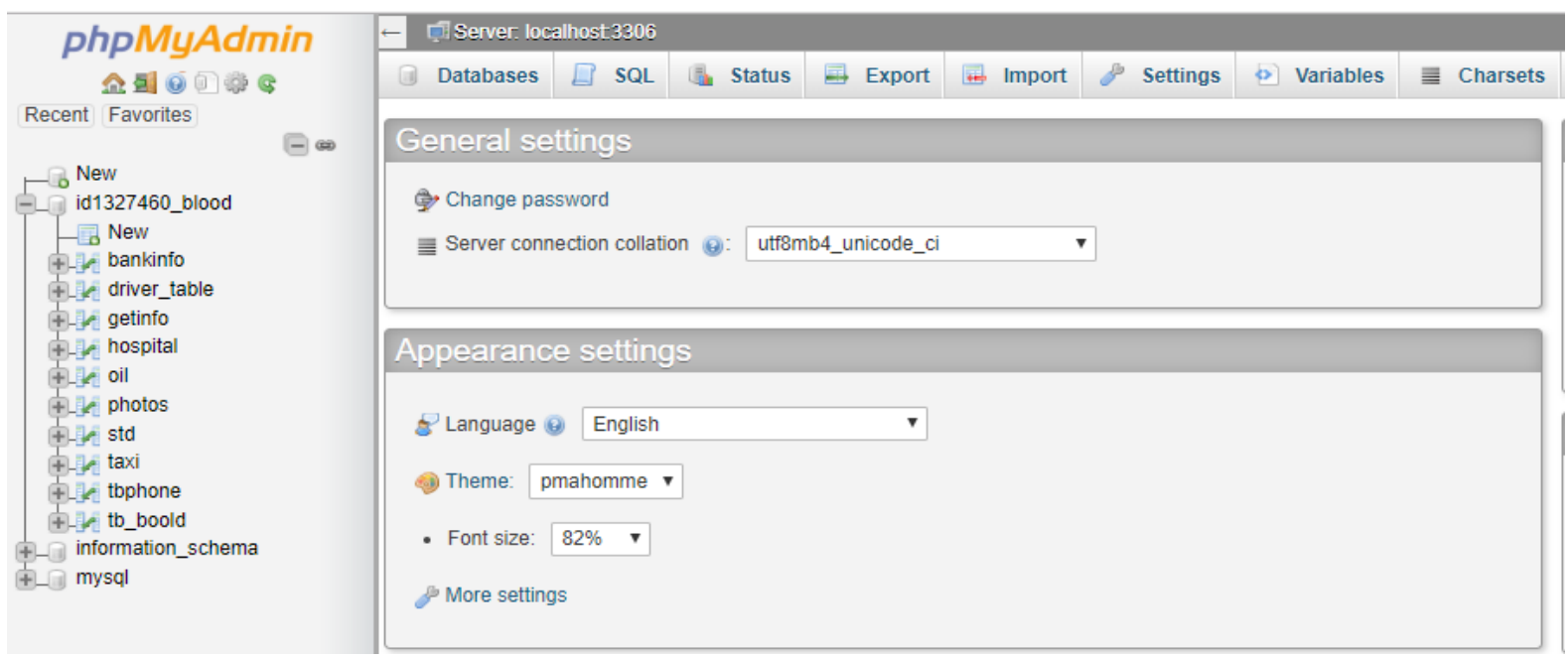

Figure 3: Database Server

The following code is connection between server and database by php. 
$<$ php ?>

\$conn = mysqli_connect("DB Host ", " DB User ", "DB Password", " DB Name");

\subsubsection{PHP}

In the server side, HTML embedded scripting language that may be used to create dynamic web pages. It is available for most operating systems and Web servers, and can access most common databases, including MySQL. PHP may be run as a separate program or compiled as a module for use with a Web server. (Baron Schwarz, Peter Zaitsev, Vadim Tkachenko, 2012)

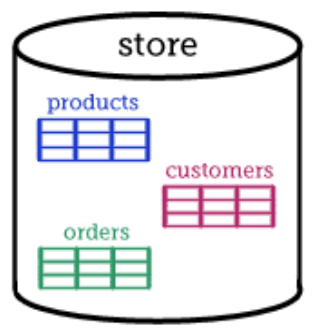

Database

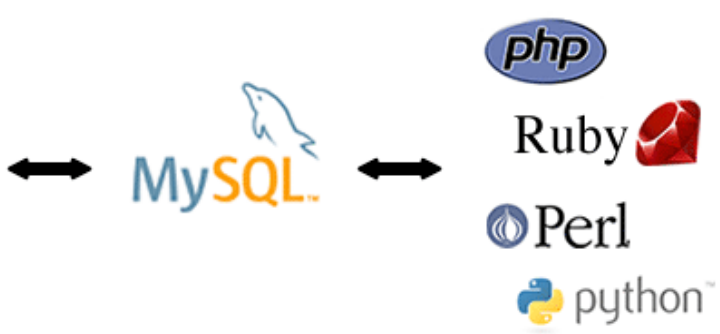

Website Code

Figure 4: Database Diagram

A database (left) is a collection of related data, managed by a DBMS such as MySQL (center). Web scripts (right) communicate with the DBMS in order to insert, update, delete and retrieve data in the database.

\subsubsection{Graphic System}

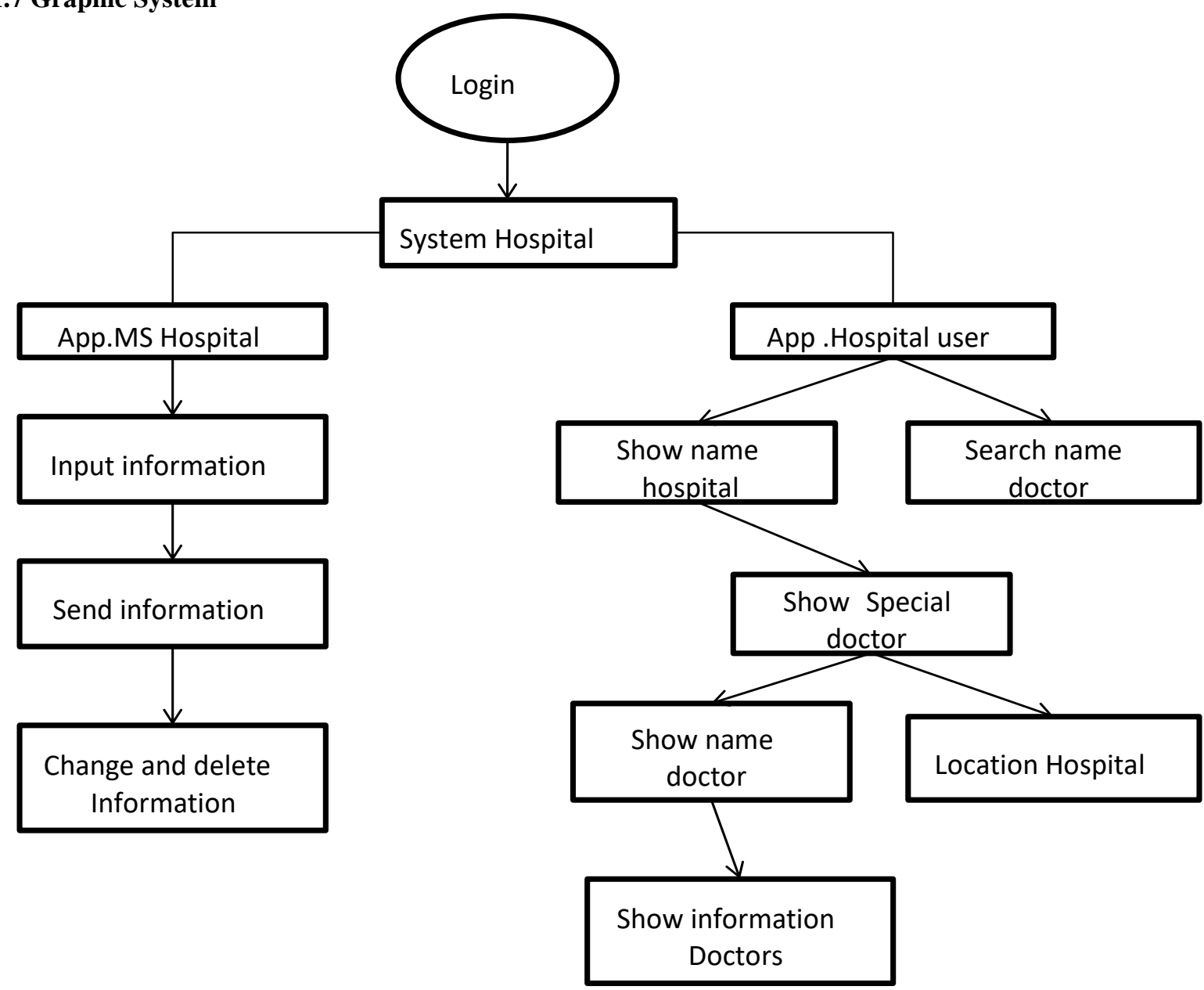




\title{
International Journal of Advanced Research in Computer and Communication Engineering
}

\author{
Vol. 7, Issue 10, October 2018
}

\subsubsection{Implementation}

System implementation is a stage in system life cycle whereby a new system is developed, installed and made ready for use. It is this stage that all details and key point in the requirement specification are divided. System implementation therefore, is a very essential stage in which its success determines to a great extent the success of the new system. At this instance, after all is said and done the system is duly ready to be implemented (Booking Taxi System). System design is concerned mainly with the coordination of activities, job procedures and equipment utilization in order to achieve organizational objectives. It addresses data input and output data, processing and interface:

\subsubsection{Name of Special Hospital}

This list is consisting of the name at a special hospital in Duhok governorate. By your desire you can choose one of these items, then show and patient all information about the hospital.

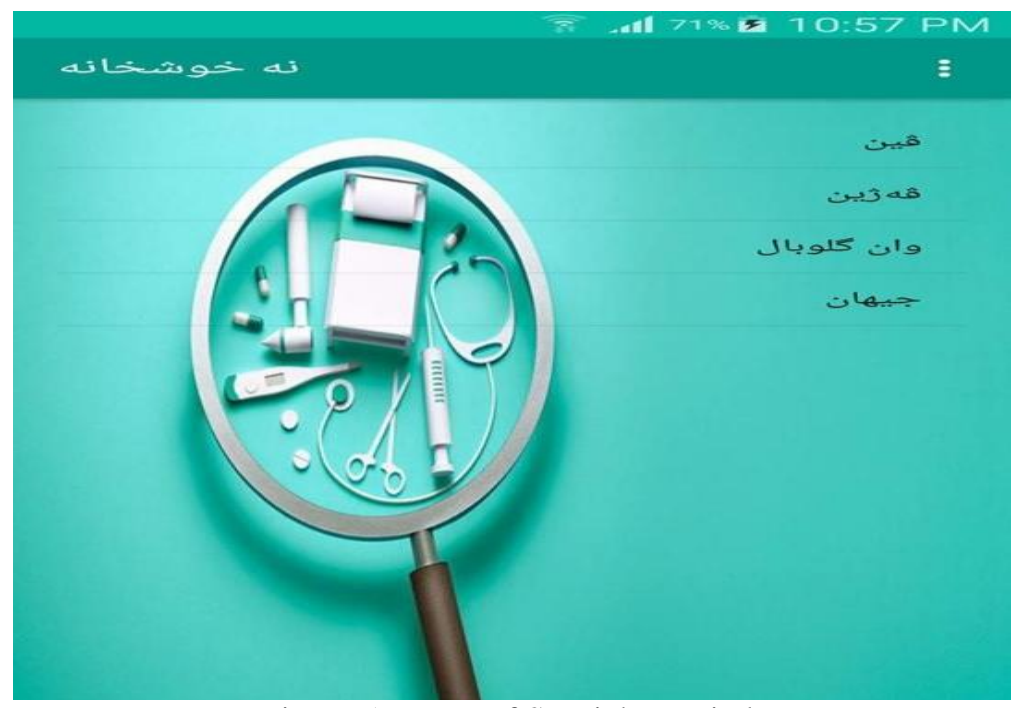

Figure 5: Name of Special Hospital

\subsubsection{Special Doctors}

This list consists of specialty doctors in that special hospital in Duhok governorate when you choose one of this specialists, it will show you the name of doctors who are in this list.

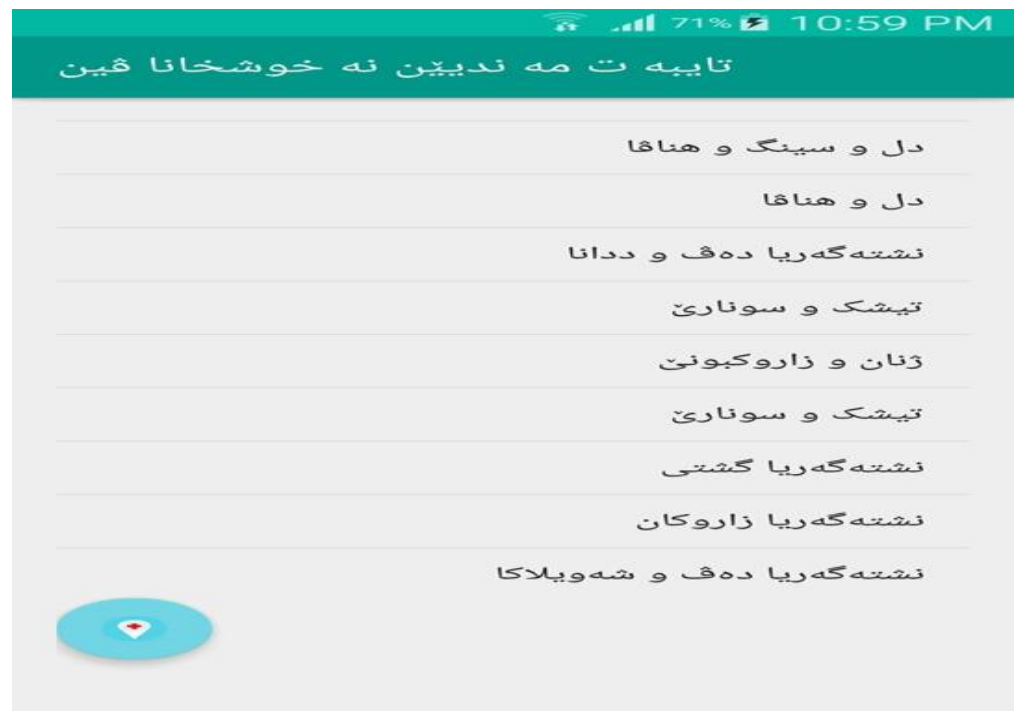

Figure 6: Special Doctors

\subsubsection{Logo Location and Hospital Location}

(Figure $7 \mathrm{a}$ and Figure $7 \mathrm{~b}$ ) This (Logo) has shown the place of hospital, if you press click on the (Logo) it will be show the place of hospital 


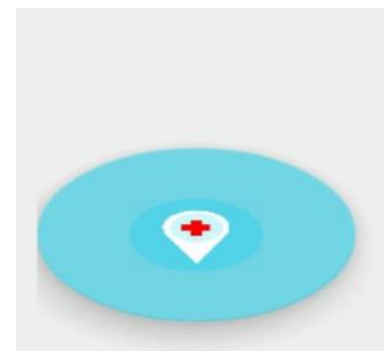

Figure 7 a: Logo Location

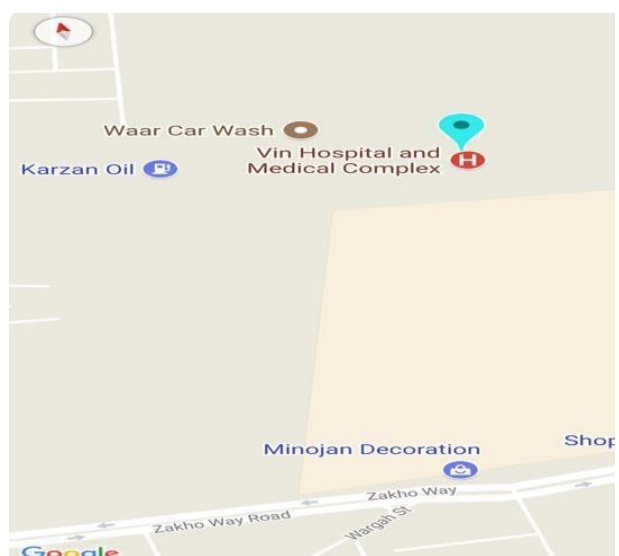

Figure 7 b: Location Hospital

\subsubsection{Names of Doctors}

This list consist the name of doctors, when we choose the specialty of doctors, and when we choose one of this name, it will show to us information about doctors. As shown in Figure 8.

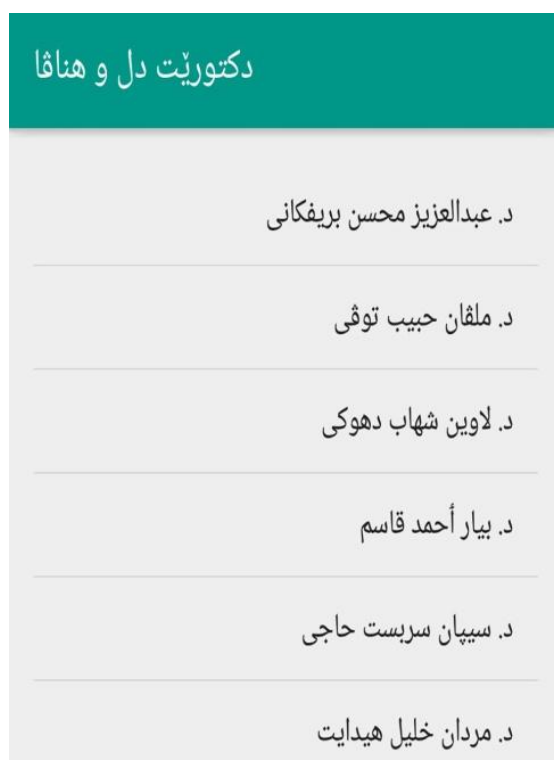

Figure 8: Names of Doctors

\subsubsection{Information of a Doctor}

This list consists of information about doctors, and this information consists of:

- $\quad$ Name of doctor

- $\quad$ Phone number

- Holiday days and evaluate the doctor too 
Vol. 7, Issue 10, October 2018

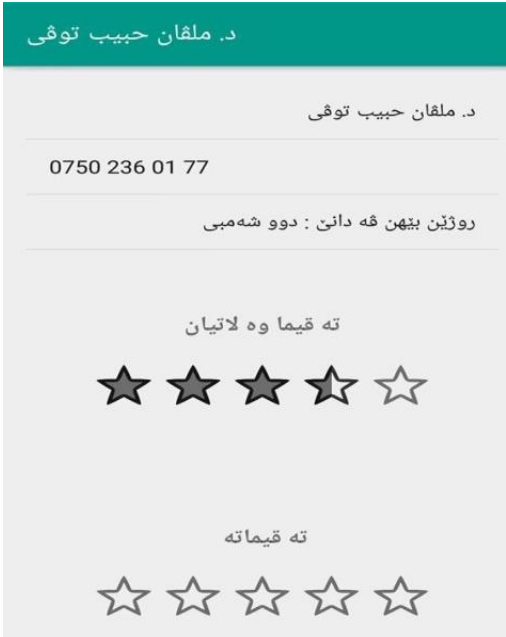

Figure 9: Information a Doctor

\subsubsection{Reserve}

When you click on the number phone you can direct calling for doctor

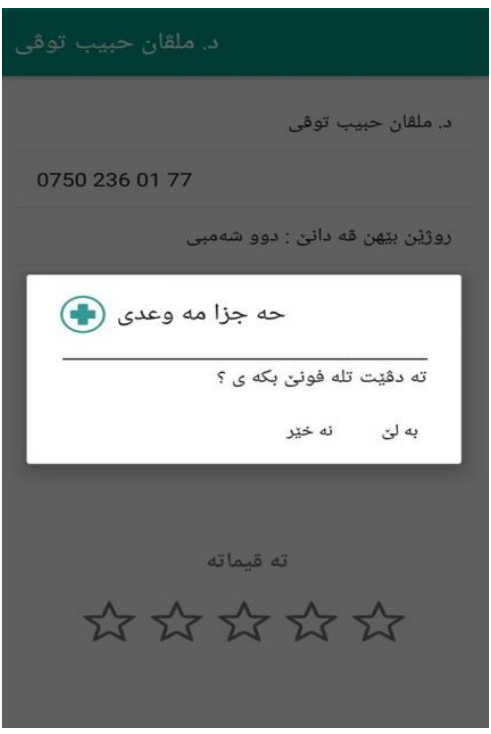

Figure 10: Reserve

\subsubsection{Search Doctor's Name}

We use (Search) for searching the name of doctor, if you know the name of doctor but when do not know the place and his hospital you can search his name, it will be shown all information about doctor.

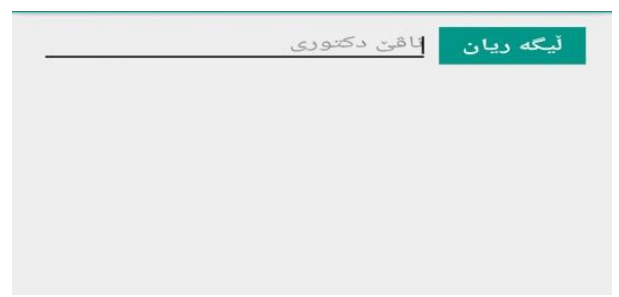

Figure 11: Search Doctor's Name

\subsubsection{Username and Password}

For input the doctor information and hospital name, each hospital management has (username and password), by this the management only can changing and delete the information about doctors. 


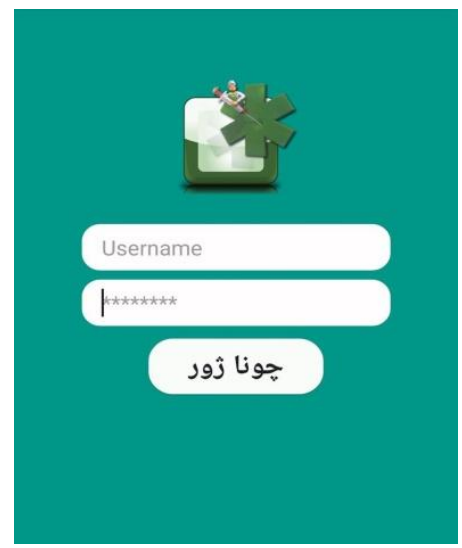

Figure 12: Username and Password

\subsubsection{Input Information of Doctor and Hospital}

This list for input the all information about doctor and hospital, consist of.

- Name of hospital

- Specialty of doctor

- Name of doctor

- $\quad$ Phone number

- Holiday day

Point location (latitude \& longitude)

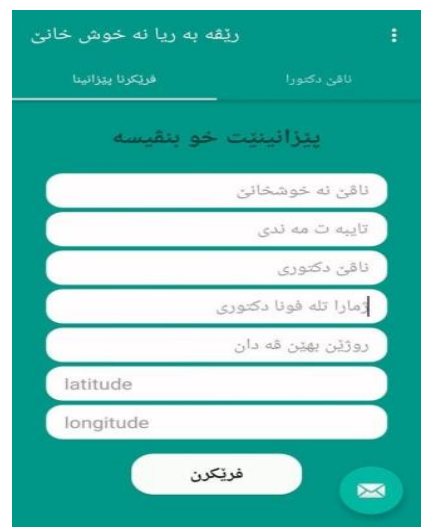

Figure 13: Input Information of Doctor and Hospital

\subsubsection{0 list names of doctors of a special hospital}

This list has names of doctors and information, each management has a specialist list used for hospital management only which can be used, and only click on the name of doctors you can change and delete information in that list for hospital management only it can be used, and only click on the name doctors you can change and delete information in that list.

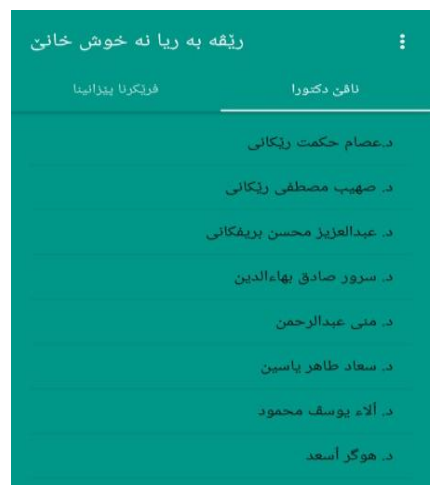

Figure 14 a: list name doctor special hospital 
Vol. 7, Issue 10, October 2018

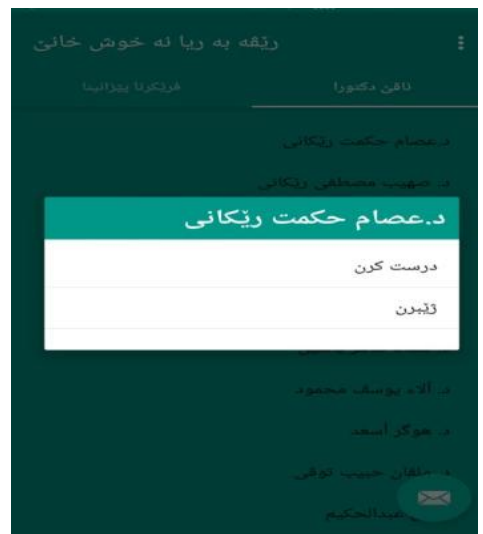

Figure 14 b: Change and Delete Information Doctor

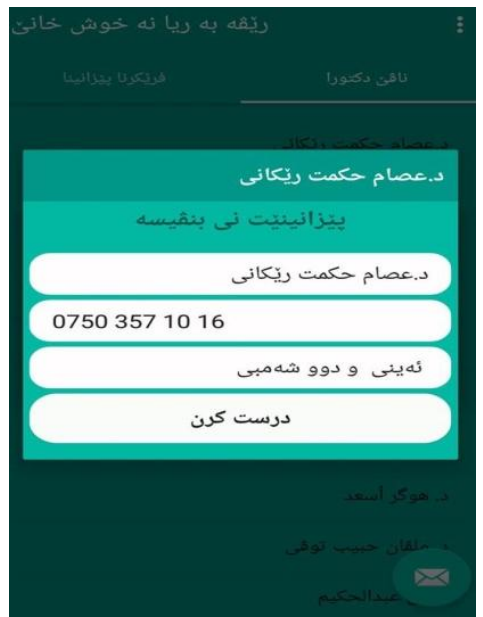

Figure 14 c: Change Information Doctor

III. CONCLUSIONS

The purpose of this application the we do service for people of Duhok governorate, and collect more information buy regular for the people can get easier information about special hospital and show the place of hospital by (GPS) and we hope this application to be service for all people of Duhok governorate, in future we are more trying for doing our ability for making better and strongest application.

\section{SUGGESTIONS FOR FUTURE WORK}

Base on the findings and conclusion of the study. The following recommendations are advice for the future researchers:

- Show the price of doctor ticket.

- Input all types of medicine and how to use.

- Input the healthy information.

- How to save yourself and solution of same type's illness.

\section{REFERENCES}

[1]. https://en.wikipedia.org/wiki/Android_(operating_system)\#History

[2]. Android Notes for professionals.

[3]. https://en.wikipedia.org/wiki/Android_Studio\#Features

[4]. Hussain Fakhruddin Top 13 New Features In Android Studio 3.0 May 18, 2017

[5]. http://teks.co.in/site/blog/top-13-new-features-in-android-studio-3-0/

[6]. High. Performance. MySQL .3rd. Edition.

[7]. Oreilly.Understanding.MyQSL.Internals.Apr.2007.

[8]. Kroenke, David 2008 Using MIS - 2nd Edition.

[9]. https://examples.javacodegeeks.com/android/core/ui/ratingbar/android-rating-barexample/\#respond

[10]. How to Create a Google Map Application using Android Studio Posted on December 31, 2013.

[11]. https://coderwall.com/p/zpwrsg/add-search-function-to-list-view-in-android 